Proyecciones Journal of Mathematics

Vol. 30, N ${ }^{o}$ 1, pp. 77-90, May 2011.

Universidad Católica del Norte

Antofagasta - Chile

\title{
Numerical range of a pair of strictly upper triangular matrices
}

\author{
Wen Yan \\ Tuskegee University, U. S. A. \\ Received : January 2011. Accepted : March 2011
}

\begin{abstract}
Given two strictly upper triangular matrices $X, Y \in C_{m \times m}$, we study the range $W_{Y}(X)=\left\{\operatorname{trn} X n^{-1} Y^{*}: n \in N\right\}$, where $N$ is the group of unit upper triangular matrices in $C_{m \times m}$. We prove that it is either a point or the whole complex plane. We characterize when it is a point.

We also obtain some convexity result for a similar range, where $N$ is replaced by any ball of $C^{k}(k=m(m-1) / 2)$ embedded in $N$, $m \leq 4$.
\end{abstract}

2000 Mathematics Subject Classification : Primary 15A60.

Key Words and Phrases : Numerical range, unit upper triangular matrices, strictly upper triangular matrices. 


\section{Introduction}

Let $C_{m \times m}$ be the space of all $m \times m$ complex matrices. The classical numerical range of $A \in C_{m \times m}$ is defined as

$$
W(A):=\left\{x^{*} A x: x^{*} x=1, x \in C^{m}\right\} \subset C .
$$

The celebrated Toeplitz-Hausdorff theorem [9] asserts that $W(A)$ is a compact convex subset of $C$. There are numerous generalizations $[5,1,4,8$, $7,10,11,12,14]$ and our references are far from complete. One important view is to deem the numerical range as the image of an orbit under the linear functional [2] determined by $A$, that is,

$$
W(A)=\left\{\operatorname{tr} A x x^{*}: x \in C^{m}, x^{*} x=1\right\} .
$$

The set

$$
\left\{x x^{*}: x \in C^{m}, x^{*} x=1\right\}=O\left(E_{11}\right):=\left\{U E_{11} U^{*}: U \in U(m)\right\}
$$

is viewed as an orbit of the matrix $E_{11}:=\operatorname{diag}(1,0, \ldots, 0)$ under the conjugation action of $U(m)$, where $U(m)$ denotes the unitary group in $C_{m \times m}$. In general, if $C \in C_{m \times m}$, then denote by

$$
O(C):=\left\{U C U^{*}: U \in U(m)\right\}
$$

the orbit of $C$ under the conjugation action of $U(n)$. The $C$-numerical range of $A[13,3]$ is defined to be the set

$$
W_{C}(A):=\{\operatorname{tr} A Y: Y \in O(C)\} .
$$

If $C=\operatorname{diag}(1, \ldots, 1,0, \ldots, 0),(k 1$ 's $)$, it becomes Halmos's $k$-numerical range [7] of $A$

$$
W_{k}(A)=\left\{\sum_{j=1}^{k} x_{j}^{*} A x_{j}: x_{1}, \ldots, x_{k} \in C^{m} \quad \text { are orthonormal }\right\} .
$$

If $C=\operatorname{diag}\left(c_{1}, \ldots, c_{m}\right)$ (c's are real), the $C$-numerical range of $A$ becomes Westwick's $c$-numerical range [14] of $A$

$$
W_{c}(A)=\left\{\sum_{j=1}^{m} c_{j} x_{j}^{*} A x_{j}: x_{1}, \ldots, x_{m} \in C^{m} \quad \text { are orthonormal }\right\} .
$$


Westwick's theorem [14] asserts that the $c$-numerical range of $A$ is convex. The orbital point of view leads to several generalizations of the numerical range. Moreover the convexity result has been successfully extended in the context of compact Lie groups [11] and most real classical semisimple Lie algebras $[8,4,12]$. Usually the groups involved in the relevant orbital generalizations are compact (for example $U(m)$ is compact in the setting of the $c$-numerical range).

In this note we consider the group of $m \times m$ unit upper triangular matrices which is non-semisimple and noncompact. By a unit upper triangular matrix, we mean an upper triangular with diagonal entries all ones. Let $N$ be the group of unit upper triangular matrices in $C_{m \times m}$. It is a unipotent (noncompact) Lie group whose Lie algebra $n$ is the set of strictly upper triangular matrices in $C_{m \times m}$. Given $X \in n$, denote by

$$
O(X):=\left\{n X n^{-1}: n \in N\right\} \subset n
$$

the orbit of $X$ under the conjugation action of the group $N$. Let $X, Y \in n$. The numerical range of the pair $(X, Y)$ is defined as

$$
W_{Y}(X):=\left\{\operatorname{tr} n X n^{-1} Y^{*}: n \in N\right\} .
$$

It may be interpreted as the image of the orbit $O(X)$ under the linear functional determined by $Y$. In Section 2 we prove that $W_{Y}(X)$ is either a point (not necessarily the origin) or $C$. In Section 3 , given $r>0, c_{i j} \in C$, $1 \leq i<j \leq m$, we consider a compact subset of $N$ :

$$
N_{1}:=\left\{n:=\left(n_{i j}\right) \in N: \sum_{1 \leq i<j \leq m}\left|n_{i j}-c_{i j}\right|^{2}=r^{2}\right\} .
$$

In other words, the ball of radius $r$ (with respect to the 2-norm) centered at $c$ of $C^{s}$ is embedded as $N_{1} \subset N$, where $s=m(m-1) / 2$. We consider the restricted range:

$$
W_{Y}^{1}(X):=\left\{\operatorname{tr} n X n^{-1} Y^{*}: n \in N_{1}\right\} .
$$

When $m=2,3,4$ we prove that $W_{Y}^{1}(X)$ is a convex set. When $m>4$ convexity of $W_{Y}^{1}(X)$ is unknown.

\section{The shape of $W_{Y}(X)$}

Theorem 1. Let $X, Y \in n$. When $m=2, W_{Y}(X)=\left\{\operatorname{tr} n X n^{-1} Y^{*}: n \in\right.$ $N\}$ is a singleton set $\{x \bar{y}\}$ if

$$
X=\left(\begin{array}{ll}
0 & x \\
0 & 0
\end{array}\right), \quad Y=\left(\begin{array}{ll}
0 & y \\
0 & 0
\end{array}\right) .
$$


When $m>2, W_{Y}(X)$ is either a point or the whole complex plane $C$. If $W_{Y}(X)$ is a point, then the point is $\sum_{1 \leq i<\ell \leq m} x_{i \ell} \bar{y}_{i \ell}$. More precisely, $W_{Y}(X)=C$ if and only if one of the following is true.

(i) $x_{j k} \bar{y}_{i \ell} \neq 0$ for some $i, j, k$ and $\ell$ such that

(a) $1 \leq i<j<k<\ell \leq m$, or

(b) $1 \leq i=j<k<\ell-1 \leq m-1$, or

(c) $2 \leq i+1<j<k=\ell \leq m$.

(ii) $x_{j k} \bar{y}_{i \ell}=0$ for all $1 \leq i<j<k<\ell \leq m$, but there exist $i, \ell$ such that $i<\ell-1, x_{i, \ell-1} \bar{y}_{i \ell} \neq 0$ and $x_{i, \ell-1} \bar{y}_{i \ell} \neq x_{\ell t} \bar{y}_{\ell-1, t}$ for all $\ell<t \leq m$, or $x_{i+1, \ell} \bar{y}_{i \ell} \neq 0$ and $x_{i+1, \ell} \bar{y}_{i \ell} \neq x_{t i} \bar{y}_{t, i+1}$ for all $1 \leq t<i$.

Proof. The case $m=2$ is trivial. Suppose $m>2$. Let $n=\left(n_{i j}\right) \in N$. Clearly $M:=n^{-1}$ is upper triangular. Because of the upper triangular form of $n, X, Y, M$, we have

$$
\operatorname{tr} n X n^{-1} Y^{*}=\sum_{1 \leq i \leq j<k \leq \ell \leq m} n_{i j} x_{j k} M_{k \ell} \bar{y}_{i \ell} .
$$

Notice that the $(k, \ell)$ entry of $M$ is

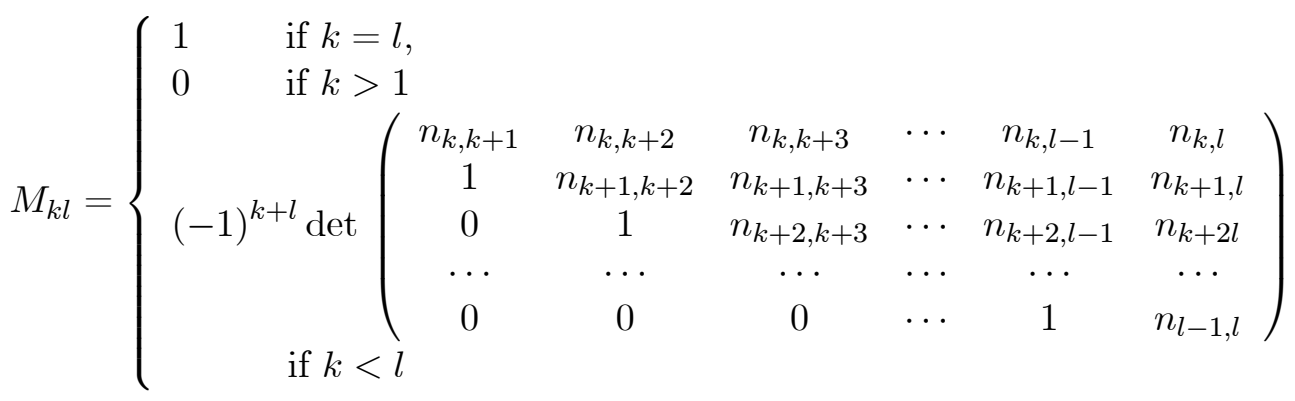

Notice that $M_{k \ell}$ is a polynomial in the variables $n_{s t}, k \leq s<t \leq \ell$. Moreover the exponent of each $n_{s t}$ in the expression (2.1) of $M_{k \ell}$ is either 0 or 1.

Evidently $\operatorname{tr} n X n^{-1} Y^{*}$ is a polynomial of $n_{i j}, 1 \leq i<j \leq m$. Since $n_{i j}$ does not appear in the polynomial $M_{k \ell}$ for $i \leq j<k \leq \ell$, the exponent of any $n_{i j}(i<j)$ in $\operatorname{tr} n X n^{-1} Y^{*}$ is either 0 or 1 . We use $n_{1}, \ldots, n_{r}$ to denote those $n_{i j}(i<j)$ which appear in the polynomial $\operatorname{tr} n X n^{-1} Y^{*}$. Let

$$
f_{0}\left(n_{1}, n_{2}, \ldots, n_{r}\right):=\operatorname{tr} n X n^{-1} Y^{*} .
$$


1. If $f_{0}$ is a constant polynomial. Then $\left\{\operatorname{tr} n X n^{-1} Y^{*}: n \in N\right\}$ is a point.

2. Otherwise, we can rewrite $f_{0}$ as

$$
f_{0}\left(n_{1}, \ldots, n_{r}\right)=n_{1} f_{1}\left(n_{2}, \ldots, n_{r}\right)+f_{2}\left(n_{2}, \ldots, n_{r}\right),
$$

where $f_{1}$ is either a nonconstant polynomial in $n_{2}, n_{3} \ldots, n_{r}$ or a nonzero constant number $c$. In either case we can choose complex numbers $c_{2}, \ldots, c_{r}$ for $n_{2}, \ldots, n_{r}$ such that $f_{1}\left(c_{2}, \ldots, c_{r}\right) \neq 0$. By the fundamental theorem of algebra $\left\{f_{0}\left(n_{1}, c_{2}, \ldots, c_{r}\right): n_{1} \in C\right\}=C$. Hence $W_{Y}(X)=C$.

So $W_{Y}(X)$ is either a point or $C$.

We are going to show that $W_{Y}(X)=C$ if either (i) or (ii) holds. Suppose (i)(a) is true, that is, there exists $x_{j_{0} k_{0}} \bar{y}_{i_{0} \ell_{0}} \neq 0$ for some $1 \leq i_{0}<$ $j_{0}<k_{0}<\ell_{0} \leq m$. Define

$$
n(s):=\left(n_{i j}\right)=I_{m}+s E_{i_{0}, j_{0}}+s E_{k_{0}, \ell_{0}} \in N, \quad s \in C,
$$

and $E_{i j}$ is the matrix with 1 as the $(i, j)$ entry and zeros elsewhere. So $M:=n(s)^{-1}=I_{m}-s E_{i_{0}, j_{0}}-s E_{k_{0}, \ell_{0}}$. Then

$$
f(s):=\operatorname{tr} n(s) X n(s)^{-1} Y^{*}=\sum_{1 \leq i \leq j<k \leq \ell \leq m} n_{i j} x_{j k} M_{k l} \bar{y}_{i \ell}
$$

is a quadratic polynomial in $s$, and the leading term of $f(s)$ is $n_{i_{0} j_{0}} x_{j_{0} k_{0}} M_{k_{0} \ell_{0}} \bar{y}_{i_{0} \ell_{0}}=$ $-x_{j_{0} k_{0}} \bar{y}_{i_{0} \ell_{0}} s^{2}$. Therefore

$$
C=\{f(s): s \in C\} \subset W_{Y}(X) \subset C .
$$

We now insert a lemma.

Lemma 2. Suppose (i)(a) is not true.

1. If there exist $1 \leq i_{0}<k_{0}<\ell_{0} \leq m$ such that $x_{i_{0} k_{0}} \bar{y}_{i_{0} \ell_{0}} \neq 0$, then $x_{i k_{0}} \bar{y}_{i \ell_{0}}=0$ for all $i \neq i_{0}$.

2. If there exist $1 \leq i_{0}<j_{0}<\ell_{0} \leq m$ such that $x_{j_{0} \ell_{0}} \bar{y}_{i_{0} \ell_{0}} \neq 0$, then $x_{j_{0} \ell} \bar{y}_{i_{0} \ell}=0$ for all $\ell \neq \ell_{0}$. 
Proof. (1) If there exists $i_{1} \neq i_{0}$ such that $1 \leq i_{1}<k_{0}$ and $x_{i_{1} k_{0}} \bar{y}_{i_{1} \ell_{0}} \neq 0$, then we have the following two cases.

(a) if $i_{0}<i_{1}$, then $x_{i_{1} k_{0}} \bar{y}_{i_{0} \ell_{0}} \neq 0$ with $1 \leq i_{0}<i_{1}<k_{0}<\ell_{0}$,

(b) if $i_{0}>i_{1}$, then $x_{i_{0} k_{0}} \bar{y}_{i_{1} \ell_{0}} \neq 0$ with $1 \leq i_{1}<i_{0}<k_{0}<\ell_{0}$.

Both are under case (i)(a). The proof of (2) is analogous.

Suppose (i)(b) is true. Let $i_{0}, j_{0}, k_{0}$ and $\ell_{0}$ be such that $1 \leq i_{0}=j_{0}<$ $k_{0}<\ell_{0}-1 \leq m-1$ and $x_{j_{0} k_{0}} \bar{y}_{i_{0} \ell_{0}}=x_{i_{0} k_{0}} \bar{y}_{i_{0} \ell_{0}} \neq 0$. Let $n(s):=\left(n_{i j}\right) \in N$ be defined as follows:

$$
n_{k, k+1}=s, \quad k=k_{0}, \ldots, \ell_{0}-1, \quad n_{i j}=0 \text { for all other } i<j .
$$

Set

$$
g(s):=\operatorname{tr} n(s) X n(s)^{-1} Y^{*}=\sum_{1 \leq i \leq j<k \leq \ell \leq m} n_{i j} x_{j k} M_{k \ell} \bar{y}_{i \ell},
$$

where $M:=n(s)^{-1}$ and

$$
M_{k l}=\left\{\begin{array}{l}
(-1)^{k+l} s^{l-k} \text { if } k_{0} \leq k \leq l_{0}, \\
1 \text { if } k=l, \\
0 \text { for all other } k, l
\end{array}\right.
$$

Notice that $\operatorname{deg} g(s)=\ell_{0}-k_{0}$. Only $M_{k_{0} \ell_{0}}=(-1)^{\ell_{0}+k_{0}} s^{\ell_{0}-k_{0}}$ of $M$ has the highest degree. Moreover $n_{i j}$ in $n_{i j} x_{j k_{0}} s^{\ell_{0}-k_{0}-1} \bar{y}_{i, \ell_{0}-1}=n_{i j} x_{j k_{0}} M_{k_{0}, \ell_{0}-1} \bar{y}_{i, \ell_{0}-1}$ $\left(i \leq j<k_{0}<\ell_{0}-1\right)$ or $n_{i j} x_{j, k_{0}+1} s^{\ell_{0}-k_{0}-1} \bar{y}_{i \ell_{0}}=n_{i j} x_{j, k_{0}+1} M_{k_{0}+1, \ell_{0}} \bar{y}_{i \ell_{0}}$ $\left(i \leq j<k_{0}+1<\ell_{0}\right)$ cannot be $s$, by (2.1). So the leading term of $g(s)$ is

$$
(-1)^{k_{0}+\ell_{0}}\left[\sum_{1 \leq i \leq j<k_{0}} n_{i j} x_{j k_{0}} \bar{y}_{i \ell_{0}}\right] s^{\ell_{0}-k_{0}}=(-1)^{k_{0}+\ell_{0}}\left[\sum_{1 \leq i<k_{0}} x_{i k_{0}} \bar{y}_{i \ell_{0}}\right] s^{\ell_{0}-k_{0}} .
$$

If (i)(a) is not true, then by Lemma 2(1), (2.2) becomes

$$
(-1)^{k_{0}+\ell_{0}} x_{i_{0} k_{0}} \bar{y}_{i_{0} \ell_{0}} s^{\ell_{0}-k_{0}} .
$$

Therefore $\{g(s): s \in C\}=C$ and hence $W_{Y}(X)=C$.

If (i)(c) is true, then there exist $2 \leq i_{0}+1<j_{0}<\ell_{0} \leq m$ such that $x_{j_{0} \ell_{0}} \bar{y}_{i_{0} \ell_{0}} \neq 0$. Let $n(s):=\left(n_{i j}\right)=I_{m}+s E_{i_{0}, j_{0}} \in N, s \in C$. Then $M:=n(s)^{-1}=I_{m}-s E_{i_{0}, j_{0}}$. We may assume that (i)(a) is not true. Then $x_{j_{0} \ell} \bar{y}_{i_{0} \ell}=0$ for all $\ell \neq \ell_{0}$ by Lemma 2(2). Thus the only possible nonconstant term in the polynomial

$$
h(s):=\operatorname{tr} n(s) X n(s)^{-1} Y^{*}=\sum_{1 \leq i \leq j<k \leq \ell \leq m} n_{i j} x_{j k} M_{k \ell} \bar{y}_{i \ell}
$$


is

$\sum_{j_{0}<\ell \leq m} n_{i_{0} j_{0}} x_{j_{0} \ell} M_{\ell \ell} \bar{y}_{i_{0} \ell}+\sum_{1 \leq i<i_{0}} n_{i i} x_{i i_{0}} M_{i_{0} j_{0}} \bar{y}_{i j_{0}}=x_{j_{0} \ell_{0}} \bar{y}_{i_{0} \ell_{0}} s-\sum_{1 \leq i<i_{0}} x_{i i_{0}} \bar{y}_{i j_{0}} s$.

Since $i_{0}+1<j_{0}$, if there exists $x_{i i_{0}} \bar{y}_{i j_{0}} \neq 0$ for some $i<i_{0}$, then this becomes case (i)(b) and $W_{Y}(X)=C$. Otherwise $x_{i i_{0}} \bar{y}_{i j_{0}}=0$ for all $1 \leq$ $i<i_{0}$, then the leading term of $h(s)$ is $x_{j_{0} \ell_{0}} \bar{y}_{i_{0} \ell_{0}} s$ with nonzero coefficient. Therefore $\{h(s): s \in C\}=C$ and hence $W_{Y}(X)=C$.

Suppose condition (ii) holds. Then there exist $1 \leq i_{0}<\ell_{0}-1 \leq m-1$ such that (1) $x_{i_{0}, \ell_{0}-1} \bar{y}_{i_{0} \ell_{0}} \neq 0$ and $x_{i_{0}, \ell_{0}-1} \bar{y}_{i_{0} \ell_{0}} \neq x_{\ell_{0} t} \bar{y}_{\ell_{0}-1, t}$ for all $t>\ell_{0}$, or $(2) x_{i_{0}+1, \ell_{0}} \bar{y}_{i_{0} \ell_{0}} \neq 0$ and $x_{i_{0}+1, \ell_{0}} \bar{y}_{i_{0} \ell_{0}} \neq x_{t_{0}} \bar{y}_{t, i_{0}+1}$ for all $1 \leq t<i_{0}$. We may assume that condition (i) does not hold.

(1) Define $n(s):=\left(n_{i j}\right)=I_{m}+s E_{\ell_{0}-1, \ell_{0}} \in N, s \in C$. So $M:=n(s)^{-1}=$ $I_{m}-s E_{\ell_{0}-1, \ell_{0}}$. Let

$$
u(s):=\operatorname{tr} n(s) X n(s)^{-1} Y^{*}=\sum_{1 \leq i \leq j<k \leq \ell \leq m} n_{i j} x_{j k} M_{k \ell} \bar{y}_{i \ell} .
$$

Then

$$
\begin{aligned}
u(s)= & \sum_{1 \leq i \leq \ell_{0}-1} n_{i i} x_{i, \ell_{0}-1} M_{\ell_{0}-1, \ell_{0}} \bar{y}_{i \ell_{0}}+\sum_{\ell_{0}<\ell \leq m} n_{\ell_{0}-1, \ell_{0}} x_{\ell_{0} \ell} M_{\ell \ell} \bar{y}_{\ell_{0}-1, \ell} \\
& +\sum_{1 \leq i<\ell \leq m} n_{i i} x_{i \ell} M_{\ell \ell} \bar{y}_{i \ell} \\
= & -x_{i_{0}, \ell_{0}-1} \bar{y}_{i_{0} \ell_{0}} s+\sum_{\ell_{0}<t \leq m} x_{\ell_{0} t} \bar{y}_{\ell_{0}-1, t} s+\sum_{1 \leq i<\ell \leq m} x_{i \ell} \bar{y}_{i \ell} .
\end{aligned}
$$

The last equality is due to Lemma 2(1) which implies $x_{i, \ell_{0}-1} \bar{y}_{i \ell_{0}}=0$ for all $i \neq i_{0}$. Therefore, if $x_{\ell_{0} t} \bar{y}_{\ell_{0}-1, t}=0$ for all $t>\ell_{0}$, then

$$
u(s)=-x_{i_{0}, \ell_{0}-1} \bar{y}_{i_{0} \ell_{0}} s+\sum_{1 \leq i<\ell \leq m} x_{i \ell} \bar{y}_{i \ell} .
$$

Otherwise by Lemma $2(2)$ there is only one $t$, say $t_{0}$, such that $x_{\ell_{0} t} \bar{y}_{\ell_{0}-1, t} \neq 0$. Hence

$$
u(s)=\left(x_{\ell_{0} t_{0}} \bar{y}_{\ell_{0}-1, t_{0}}-x_{i_{0}, \ell_{0}-1} \bar{y}_{i_{0} \ell_{0}}\right) s+\sum_{1 \leq i<\ell \leq m} x_{i \ell} \bar{y}_{i \ell},
$$

where $x_{i_{0}, \ell_{0}-1} \bar{y}_{i_{0} \ell_{0}} \neq x_{\ell_{0} t_{0}} \bar{y}_{\ell_{0}-1, t_{0}}$ by (ii). In both cases, the polynomial $u(s)$ is linear. Thus $W_{Y}(X)=C$. 
(2) If there exist $x_{i_{0}+1 \ell_{0}} \bar{y}_{i_{0} \ell_{0}} \neq 0$ with $1 \leq i_{0}<\ell_{0}-1 \leq m-1$ and $x_{i_{0}+1 \ell_{0}} \bar{y}_{i_{0} \ell_{0}} \neq x_{t i_{0}} \bar{y}_{t i_{0}+1}$ for all $1 \leq t<i_{0}$.

Define $n(s):=\left(n_{i j}\right)=I_{m}+s E_{i_{0}, i_{0}+1} \in N, s \in C$. Then $M:=$ $n(s)^{-1}=I_{m}-s E_{i_{0}, i_{0}+1}$. Let

$$
v(s):=\operatorname{tr} n(s) X n(s)^{-1} Y^{*} .
$$

By Lemma $2(2), x_{i_{0}+1, \ell} \bar{y}_{i_{0} \ell}=0$ for all $\ell \neq \ell_{0}$. Thus

$$
\begin{aligned}
v(s)= & \sum_{i_{0}+1<\ell \leq m} n_{i_{0}, i_{0}+1} x_{i_{0}+1, \ell} M_{\ell \ell} \bar{y}_{i_{0} \ell}+\sum_{1 \leq i<i_{0}} n_{i i} x_{i i_{0}} M_{i_{0}, i_{0}+1} \bar{y}_{i, i_{0}+1} \\
& +\sum_{1 \leq i \leq \ell \leq m} x_{i \ell} M_{\ell \ell} \bar{y}_{i \ell} \\
= & x_{i_{0}+1, \ell} \bar{y}_{i_{0} \ell_{0}} s-\sum_{1 \leq t<i_{0}} x_{t i_{0}} \bar{y}_{t, i_{0}+1} s+\sum_{1 \leq i \leq \ell \leq m} x_{i \ell} \bar{y}_{i \ell} .
\end{aligned}
$$

Therefore, if $x_{t i_{0}} \bar{y}_{t, i_{0}+1}=0$ for all $t<i_{0}$, then

$$
v(s)=x_{i_{0}+1, \ell_{0}} \bar{y}_{i_{0} \ell_{0}} s+\sum_{1 \leq i \leq \ell \leq m} x_{i \ell} \bar{y}_{i \ell} .
$$

Otherwise by Lemma 2(1), there is only one $t$, denoted by $t_{0}$, such that $x_{t i i_{0}} \bar{y}_{t, i_{0}+1} \neq 0$. Hence

$$
v(s)=\left(x_{i_{0}+1, \ell_{0}} \bar{y}_{i_{0} \ell_{0}}-x_{t_{0} i_{0}} \bar{y}_{t_{0}, i_{0}+1}\right) s+\sum_{1 \leq i \leq \ell \leq m} x_{i \ell} \bar{y}_{i \ell},
$$

where $x_{i_{0}+1, \ell_{0}} \bar{y}_{i_{0} \ell_{0}} \neq x_{t_{0} i_{0}} \bar{y}_{t_{0}, i_{0}+1}$ by (ii). In both cases, the polynomial $v(s)$ is linear. Therefore $W_{Y}(X)=C$.

So either (i) or (ii) implies $W_{Y}(X)=C$.

Suppose (i) and(ii) are not true. Then the only nonzero terms among $x_{j k} \bar{y}_{i \ell}, 1 \leq i \leq j<k \leq \ell \leq m$, are (1) $x_{i, \ell-1} \bar{y}_{i \ell}$ with $x_{i, \ell-1} \bar{y}_{i \ell}=x_{\ell t} \bar{y}_{\ell-1, t} \neq$ 0 for some $t>\ell$, and $(2) x_{i+1, \ell} \bar{y}_{i \ell}$ with $x_{i+1, \ell} \bar{y}_{i \ell}=x_{t i} \bar{y}_{t, i+1} \neq 0$ for some $t<i$. Indeed for each case $t$ is unique by Lemma 2 . Thus

$$
\begin{aligned}
= & \sum_{1 \leq i \leq j<k \leq \ell \leq m}^{\operatorname{tr} n X n^{-1} Y^{*}} n_{i j} x_{j k} M_{k \ell} \bar{y}_{i \ell} \\
= & \sum_{1 \leq i<\ell-1 \leq m-1} n_{i i} x_{i, \ell-1} M_{\ell-1, \ell} \bar{y}_{i \ell}+\sum_{\substack{1 \leq i<\ell-1 \leq m-1 \\
\text { (since (i) does not hold) }}} n_{i, i+1} x_{i+1, \ell} M_{\ell \ell} \bar{y}_{i \ell} \\
& \quad+\sum_{1 \leq i<\ell \leq m} n_{i i} x_{i \ell} M_{\ell \ell} \bar{y}_{i \ell} \quad
\end{aligned}
$$




$$
\begin{aligned}
= & \sum_{1 \leq i<\ell-1 \leq m-1}-n_{\ell-1, \ell} x_{i, \ell-1} \bar{y}_{i \ell}+\sum_{1 \leq i<\ell-1 \leq m-1} n_{i, i+1} x_{i+1, \ell} \bar{y}_{i \ell} \\
& +\sum_{1 \leq i<\ell \leq m} x_{i \ell} \bar{y}_{i \ell} \quad\left(\text { since } M_{\ell-1, \ell}=-n_{\ell-1, \ell}\right) \\
= & \sum_{\substack{1 \leq i<\ell-1<t-1 \leq m-1\\
}}\left[\sum_{1 \leq i<\ell \leq m} x_{i \ell} \bar{y}_{i \ell} \quad\right. \text { (since (ii) does not hold) } \\
= & \sum_{1 \leq i<\ell \leq m} x_{i \ell} \bar{y}_{i \ell} .
\end{aligned}
$$

Therefore $W_{Y}(X)=\left\{\sum_{1 \leq i<\ell \leq m} x_{i \ell} \bar{y}_{i \ell}\right\}$.

\section{Convexity of $W_{Y}^{1}(X)$}

Given $c_{i j} \in C, 1 \leq i<j \leq m, r>0$, let

$$
N_{1}:=\left\{n:=\left(n_{i j}\right) \in N: \sum_{1 \leq i<j \leq m}\left|n_{i j}-c_{i j}\right|^{2}=r^{2}\right\} \subset N .
$$

In other words, $N_{1}$ is the embedding in $N$ of the ball in $C^{s}(s=m(m-$ 1)/2) of radius $r$ centered at $c=\left(c_{12}, \ldots, c_{1 n}, c_{23}, \ldots, c_{2 n}, \ldots, c_{n-1, n}\right)^{T}$. We define the range:

$$
W_{Y}^{1}(X):=\left\{\operatorname{tr} n X n^{-1} Y^{*}: n \in N_{1}\right\} \subset W_{Y}(X) .
$$

Theorem 1. 1. When $m=2, W_{Y}^{1}(X)$ is the singleton set $\{x \bar{y}\}$ if

$$
X=\left(\begin{array}{ll}
0 & x \\
0 & 0
\end{array}\right), \quad Y=\left(\begin{array}{ll}
0 & y \\
0 & 0
\end{array}\right)
$$

2. When $m=3$, for any $r>0, c_{1}:=c_{12}, c_{2}:=c_{13}, c_{3}:=c_{23} \in C, 1 \leq$ $i<j \leq m, W_{Y}^{1}(X)$ is the circular disc in $C$ centered at $\sum_{i=1}^{3} x_{i} \bar{y}_{i}+$ $x_{3} \bar{y}_{2} c_{1}-x_{1} \bar{y}_{2} c_{3}$ with radius $r\left|y_{2}\right| \sqrt{\left|x_{1}\right|^{2}+\left|x_{3}\right|^{2}}$, if

$$
X=\left(\begin{array}{ccc}
0 & x_{1} & x_{2} \\
0 & 0 & x_{3} \\
0 & 0 & 0
\end{array}\right), \quad Y=\left(\begin{array}{ccc}
0 & y_{1} & y_{2} \\
0 & 0 & y_{3} \\
0 & 0 & 0
\end{array}\right) \in \mathbf{n}
$$


Proof. The first statement is trivial. When $m=3$, let

$$
n=\left(\begin{array}{ccc}
1 & n_{1} & n_{2} \\
0 & 1 & n_{3} \\
0 & 0 & 1
\end{array}\right) \in N, \quad \text { such that } \sum_{j=1}^{3}\left|n_{j}-c_{j}\right|^{2}=r^{2}
$$

By direct computation

$$
\begin{aligned}
\operatorname{tr} n X n^{-1} Y^{*} & =\sum_{i=1}^{3} x_{i} \bar{y}_{i}+x_{3} \bar{y}_{2} n_{1}-x_{1} \bar{y}_{2} n_{3} \\
& =\sum_{i=1}^{3} x_{i} \bar{y}_{i}+x_{3} \bar{y}_{2} c_{1}-x_{1} \bar{y}_{2} c_{3}+x_{3} \bar{y}_{2}\left(n_{1}-c_{1}\right)-x_{1} \bar{y}_{2}\left(n_{3}-c_{3}\right) .
\end{aligned}
$$

The locus of $x_{3} \bar{y}_{2}\left(n_{1}-c_{1}\right)-x_{1} \bar{y}_{2}\left(n_{3}-c_{3}\right)$, as $n$ runs through $N_{1}$, is

$$
L=\left\{r\left(\left|x_{3} \bar{y}_{2}\right| e^{i \xi_{1}} \cos \theta+\left|x_{1} \bar{y}_{2}\right| e^{i \xi_{2}} \sin \theta\right): \theta, \xi_{1}, \xi_{2} \in[0, \pi]\right\} .
$$

It is the circular disc centered at the origin with radius $r \sqrt{\left|x_{3} \bar{y}_{2}\right|^{2}+\left|x_{1} \bar{y}_{2}\right|^{2}}$.

To establish the $4 \times 4$ case, we need the following result of Gutiérrez and Medrano [6] which generalizes the Toeplitz-Hausdorff's theorem.

Theorem 2. [6] Let $A \in C_{m \times m}$ with $m \geq 2$. Given $\alpha, \beta, c \in C^{m}$, and $r>0$. The set

$$
\left\{z^{*} A z+\alpha^{*} z+z^{*} \beta: z \in C^{m},(z-c)^{*}(z-c)=r^{2}\right\}
$$

is a compact convex set in $C$.

Theorem 3. When $m=4$, for any $r>0, c_{i j} \in C, 1 \leq i<j \leq m, W_{Y}^{1}(X)$ is a compact convex subset of $C$. In general it is not necessary a circular disk.

Proof. Let

$$
n=\left(\begin{array}{cccc}
1 & n_{1} & n_{2} & n_{3} \\
0 & 1 & n_{4} & n_{5} \\
0 & 0 & 1 & n_{6} \\
0 & 0 & 0 & 1
\end{array}\right) \in N_{1}
$$


Let

$$
X=\left(\begin{array}{cccc}
0 & x_{1} & x_{2} & x_{3} \\
0 & 0 & x_{4} & x_{5} \\
0 & 0 & 0 & x_{6} \\
0 & 0 & 0 & 0
\end{array}\right), \quad y=\left(\begin{array}{cccc}
0 & y_{1} & y_{2} & y_{3} \\
0 & 0 & y_{4} & y_{5} \\
0 & 0 & 0 & y_{6} \\
0 & 0 & 0 & 0
\end{array}\right) \in \mathbf{n}
$$

By direct computation

$$
n^{-1}=\left(\begin{array}{cccc}
1 & -n_{1} & n_{1} n_{4}-n_{2} & -n_{1} n_{4} n_{6}+n_{1} n_{5}+n_{2} n_{6}-n_{3} \\
0 & 1 & -n_{4} & n_{4} n_{6}-n_{5} \\
0 & 0 & 1 & -n_{6} \\
0 & 0 & 0 & 1
\end{array}\right)
$$

Hence

$n X n^{-1}$

$$
=\left(\begin{array}{cccc}
0 & x_{1} & x_{2}+x_{4} n_{1}-x_{1} n_{4} & x_{3}-x_{4} n_{1} n_{6}+x_{1} n_{4} n_{6}+x_{5} n_{1}+x_{6} n_{2}-x_{1} n_{5}-x_{2} n_{6} \\
0 & 0 & x_{4} & x_{5}+x_{6} n_{4}-x_{4} n_{6} \\
0 & 0 & 0 & x_{6} \\
0 & 0 & 0 & 0
\end{array}\right) .
$$

Then

$$
\begin{gathered}
\operatorname{tr} n X n^{-1} Y^{*}=\sum_{i=1}^{6} x_{i} \bar{y}_{i}-x_{4} \bar{y}_{3} n_{1} n_{6}+x_{1} \bar{y}_{3} n_{4} n_{6}+\left(x_{4} \bar{y}_{2}+x_{5} \bar{y}_{3}\right) n_{1}+x_{6} \bar{y}_{3} n_{2} \\
+(3.1) \\
+\left(x_{6} \bar{y}_{5}-x_{1} \bar{y}_{2}\right) n_{4}-x_{1} \bar{y}_{3} n_{5}-\left(x_{2} \bar{y}_{3}+x_{4} \bar{y}_{5}\right) n_{6} .
\end{gathered}
$$

Set

$$
z:=\left(n_{1}, n_{2}, n_{3}, n_{4}, n_{5}, \bar{n}_{6}\right)^{*} .
$$

Set $A:=\left(a_{i j}\right)$, where $a_{16}=-x_{4} \bar{y}_{3}, a_{46}=x_{1} \bar{y}_{3}$, and $a_{i j}=0$ otherwise.

Set

$$
\alpha:=\left(0,0,0,0,0,-\left(x_{2} \bar{y}_{3}+x_{4} \bar{y}_{5}\right)\right)^{*},
$$

and

$$
\beta:=\left(x_{4} \bar{y}_{2}+x_{5} \bar{y}_{3}, x_{6} \bar{y}_{3}, 0, x_{6} \bar{y}_{5}-x_{1} \bar{y}_{2},-x_{1} \bar{y}_{3}, 0\right)^{T} .
$$

Note that $\operatorname{tr} n X n^{-1} Y^{*}=z^{*} A z+\alpha^{*} z+z^{*} \beta$. Now

$$
W_{Y}^{1}(X)=\left\{z^{*} A z+\alpha^{*} z+z^{*} \beta: z \in C^{6},(z-c)^{*}(z-c)=r^{2}\right\} .
$$

By Theorem 2, it is convex. 
Choose $4 \times 4$ strictly upper triangular matrices $X, Y$ such that $x_{1}=$ $x_{6}=0$ and $-x_{4} \bar{y}_{3}=x_{4} \bar{y}_{2}+x_{5} \bar{y}_{3}=-\left(x_{2} \bar{y}_{3}+x_{4} \bar{y}_{5}\right)=1$. Set $c=0$. So

$$
W_{Y}^{1}(X)=\sum_{i=1}^{6} x_{i} \bar{y}_{i}+S
$$

where $S=\left\{\xi_{1}+\xi_{2}+\xi_{1} \xi_{2}: \xi_{1}, \xi_{2} \in C,\left|\xi_{1}\right|^{2}+\left|\xi_{2}\right|^{2} \leq 1\right\}$. The set $S$ is symmetric about the $x$-axis. By direct computation $S \cap R=\left[-1, \sqrt{2}+\frac{1}{2}\right]$. The set $S$ is not a circular disk by considering the point $\sqrt{2} i-\frac{1}{2} \in S$ given by $\xi_{1}=\xi_{2}=i / \sqrt{2}$.

If one replaces the expression in Theorem 2 by the form $z^{T} A z+\alpha^{T} z+$ $z^{T} \beta$ (clearly (3.1) is of this form), we may not have a convex set.

Example 4. Let $f(u)=u^{2}+2 u+1, u \in C$. If

$$
A=\operatorname{diag}(1,0, \ldots, 0) \in C_{m \times m}, \quad \alpha=(2,0, \ldots, 0)^{T}, \beta=(0, \ldots, 0)^{T} \in C^{m},
$$

the set $W:=\left\{z^{T} A z+\alpha^{T} z+z^{T} \beta+1: z \in C^{m}, z^{*} z=1\right\}=\{f(u): u \in$ $\left.C, u^{*} u=1\right\}$ is not convex.

Proof. Let $u=(\cos \theta+i \sin \theta)$, and $-\pi \leq \theta<\pi$. Then the elements of $W$ are of the form

$$
f(u)=\cos 2 \theta+2 \cos \theta+1+i(\sin 2 \theta+2 \sin \theta) .
$$

Clearly $W$ is symmetric about the $x$-axis. By choosing $\theta=-2 \pi / 3$ and $2 \pi / 3$ respectively, we have $P_{1}=-1 / 2+i \sqrt{3} / 2, P_{2}=-1 / 2-i \sqrt{3} / 2 \in W$. The midpoint $-1 / 2$ of $P_{1}$ and $P_{2}$ is not contained in $W$. Therefore $W$ is not convex.

\section{References}

[1] Y. H. Au-Yeung and N. K. Tsing, Some theorems on the generalized numerical range, Linear and Multilinear Algebra, 15, pp. 3-11, (1984).

[2] C. Davis, The Toeplitz-Hausdorff theorem explained, Canad. Math. Bull., 14, pp. 245-246, (1971). 
[3] W. S. Cheung and N. K. Tsing, The $C$-numerical range of matrices is star-shaped, Linear and Multilinear Algebra, 41, pp. 245-250, (1996).

[4] D. Z. Djokovic and T. Y. Tam, Some questions about semisimple Lie groups originating in matrix theory, Bull. Canad. Math. Soc., 46, pp. 332-343, (2003).

[5] K. R. Gustafson and D. K. M. Rao, Numerical Range: The Field of Values of Linear Operators and Matrices, Universitext, SpringerVerlag, New York, (1997).

[6] V. G. Gutiérrez and S. L. de Medrano, An extension of the ToeplitzHausdorff theorem, Bol. Soc. Mat. Mexicana (3), 9, pp. 273-278, (2003).

[7] P. R. Halmos, A Hilbert Space Problem Book, Springer-Verlag, New York, (1978).

[8] C. K. Li and T. Y. Tam, Numerical ranges arising from simple Lie algebras, J. Canad. Math. Soc., 52, pp. 141-171, (2000),

[9] R. Raghavendran, Toeplitz-Hausdorff theorem on numerical ranges, Proc. Amer. Math. Soc., 20, pp. 284-285, (1969).

[10] T. Y. Tam, An extension of a convexity theorem of the generalized numerical range associated with $S O(2 n+1)$, Proc. Amer. Math. Soc., 127, pp. 35-44, (1999).

[11] T. Y. Tam, Convexity of generalized numerical range associated with a compact Lie group, J. Austral. Math. Soc., 70, pp. 57-66, (2002).

[12] T. Y. Tam, On the shape of numerical range associated with Lie groups, Taiwanese J. Math., 5, pp. 497-506, (2001).

[13] N. K. Tsing, On the shape of the generalized numerical ranges, Linear and Multilinear Algebra, 10, pp. 173-182, (1981).

[14] R. Westwick, A theorem on numerical range, Linear and Multilinear Algebra, 2, pp. 311-315, (1975). 


\section{Wen Yan}

Department of Mathematics, Tuskegee University,

AL 36088

U. S. A.

e-mail : wyliamg@gmail.com 